\title{
COMPONENTE ARBÓREO, ESTRUTURA FITOSSOCIOLÓGICA E RELAÇÕES AMBIENTAIS EM UM REMANESCENTE DE CERRADÃO, EM CURVELO - MG
}

\author{
Thiago José Ornelas Otoni ${ }^{1}$, Israel Marinho Pereira ${ }^{2}$, Márcio Leles Romarco de Oliveira ${ }^{3}$, \\ Evandro Luiz Mendonça Machado², Múcio Magno Farnezi ${ }^{4}$, Sílvia da Luz Lima Mota ${ }^{5}$
}

(recebido: 3 de março de 2011; aceito: 25 de janeiro de 2013)

\begin{abstract}
RESUMO: Neste trabalho, objetivou-se verificar a existência de variações na composição e distribuição das espécies arbóreas em virtude das características do solo, em um fragmento de cerradão distrófico, no município de Curvelo-MG. Para a descrição da comunidade arbórea, foram instaladas dez parcelas permanentes de $1.000 \mathrm{~m}^{2}(20 \times 50 \mathrm{~m})$ com distâncias fixas de $100 \mathrm{~m}$ entre cada parcela. Todos os indivíduos arbóreos vivos com DAS (diâmetro a $0,3 \mathrm{~m}$ do nível do solo) $\geq 5,0 \mathrm{~cm}$ foram amostrados. Os indivíduos de fuste bifurcado foram incluídos quando o valor dos DAS fundidos atendia ao critério. Foram coletadas amostras de solo em duas profundidades (0-20 e 20-40 cm) e medida as distâncias das parcelas a um curso de água. Para estudar a comunidade, foram gerados diagramas de ordenação de parcelas, espécies e variáveis ambientais por meio da análise de correspondência canônica (CCA). A riqueza amostrada foi de 92 espécies, 36 famílias e 74 gêneros, destacando Erythroxylum, Byrsonima, Myrcia e Qualea. Os estimadores de jackknife (primeira e segunda ordem) projetaram uma riqueza de 106,4 e 107,8 espécies. As espécies Magonia pubescens, Terminalia argentea, Annona crassiflora, Eugenia dysenterica e Xylopia aromatica apresentaram-se distribuídas sob gradiente em função de variáveis ambientais.
\end{abstract}

Palavras-chave: Variação ambiental, cerrado, comunidade arbórea, riqueza, diversidade.

\section{ARBOREAL COMPONENT, PHYTOSOCIOLOGY STRUCTURE AND ENVIRONMENTAL RELATIONS IN A REMNANT OF CERRADÃO, CURVELO - MG}

\begin{abstract}
This work aimed to verify the existence of variations in composition and distribution of tree species due to soil characteristics in a fragment of dystrophic savanna in Curvelo-MG. On description in the compartment of the phanerophytes of this plant community, were installed ten permanent plots with $1.000 \mathrm{~m}^{2}(20 \times 50 \mathrm{~m})$ with fixed distances of $100 \mathrm{~m}$ between each plot. All alive individuals that had diameter at soil height (DSH - diameter to $0.3 \mathrm{~m}$ from ground level) $\geq 5.0 \mathrm{~cm}$ were sampled. The Individuals with multiple boles were sampled when the value of square root of the sum-square of DSH had been greater than criterion of the inventory. Were collected Soil samples at two depths $(0-20$ e 20-40 cm) and was measured the distances of plots of a watercourse. To study the community were generated ordination diagrams of plots, species and environmental variables by means of a canonical correspondence analysis (CCA). The richness sampled was 92 species, 36 families and 74 genera, highlighting Erythroxylum, Byrsonima, Myrcia and Qualea. Jackknife estimators (first and second order) designed a wealth of 106.4 and 107.8 species. The species Magonia pubescens, Terminalia argentea, Annona crassiflora, Eugenia dysenterica e Xylopia aromatica were distribuited underneath the environmental gradient.
\end{abstract}

Key-words: Environmental variation, cerrado, arboreal community, richness, diversity.

\section{INTRODUÇÃO}

O bioma Cerrado sofreu nos últimos 35 anos uma abrupta modificação em sua área original que representava 1/5 do território nacional (DURIGAN; RATTER, 2006), acarretando em uma expressiva depleção de biodiversidade perante a exploração recente dessa região para a agricultura intensiva. Esse bioma apresenta um tipo de vegetação associada a condições ecológicas especiais em que as formações abertas são predominantes, mas não exclusivas,

\footnotetext{
${ }^{1}$ Engenheiro Florestal, Mestre em Ciência Florestal - Universidade Federal dos Vales do Jequitinhonha e Mucuri/UFVJM - Departamento de Engenharia Florestal/DEF - Campus JK - Rodovia MGT 367, km 583, nº 5000 - Alto da Jacuba - 39.100-000 - Diamantina, MG, Brasil - tj.otoni@gmail.com ${ }^{2}$ Engenheiro Florestal, Professor Doutor em Engenharia Florestal - Universidade Federal dos Vales do Jequitinhonha e Mucuri/UFVJM - Departamento de Engenharia Florestal/DEF - Campus JK - Rodovia MGT 367, km 583, no 5000 - Alto da Jacuba - 39.100-000 - Diamantina, MG, Brasil imarinhopereira@gmail.com, machadoelm@gmail.com

${ }^{3}$ Engenheiro Florestal, Professor Doutor em Ciência Florestal - Universidade Federal dos Vales do Jequitinhonha e Mucuri/UFVJM - Departamento de Engenharia Florestal/DEF - Campus JK - Rodovia MGT 367, km 583, $\mathrm{n}^{\circ} 5000$ - Alto da Jacuba - 39.100-000 - Diamantina, MG, Brasil marcioromarco@gmail.com

${ }^{4}$ Engenheiro Agrônomo, Mestre em Produção Vegetal - Universidade Federal dos Vales do Jequitinhonha e Mucuri/UFVJM - Departamento de Agronomia/DAG - Campus JK - Rodovia MGT 367, km 583, nº 5000 - Alto da Jacuba - 39.100-000 - Diamantina, MG, Brasil muciomagno@yahoo.com.br

${ }^{5}$ Engenheira Florestal, Doutoranda em Ciência Florestal - Universidade de Brasília/UnB - Departamento de Engenharia Florestal - Cx. P. 04357 70919-970 - Brasília, DF, Brasil - silvialimamota@hotmail.com
}

Cerne, Lavras, v. 19, n. 2, p. 201-211, abr./jun. 2013 
compondo, assim, um mosaico de fitofisionomias alternadas entre cerrado stricto sensu, cerradões, matas ciliares ou de galeria, manchas de floresta estacional semidecidual e outras (BOURLIÈRE; HADLEY, 1983).

Muitos autores têm mostrado que comunidades vegetais de tipologias de Cerrado apresentam uma estrutura relacionada aos padrões ambientais, delimitada na maioria das vezes por fronteiras que se caracterizam ora por ecocline ora por ecótone. Mas, a maioria de suas fitofisionomias se apresenta distribuída em gradientes (COUTINHO, 1978). Oliveira Filho e Ratter (2002) salientam que o clima, o solo e a influência de incêndios florestais estão entre os fatores ambientais que melhor explicam a determinação do gradiente de vegetação de Cerrado.

Eiten (1982) sugere que a floresta cerradão está mais provável de se estabelecer em solos com maior capacidade de retenção de água. Durigan e Ratter (2006), pesquisando comunidades de Cerrado no Estado de São Paulo, descrevem a dinâmica temporal dessas áreas e atribuem a expansão do cerradão sobre as demais fitofisionomias abertas à redução das pressões antrópicas, principalmente a supressão do fogo. Além disso, mencionam a melhoria concomitante da qualidade do solo nas camadas superiores, caracterizando séries transicionais da vegetação. Essas considerações de Durigan e Ratter (2006) sugerem transformações de nicho ao longo do eixo temporal, fazendo das relações ambiente/vegetação uma série móvel que exige uma combinação com condições de não-equilíbrio.

As transições entre tipologias florestais de Cerrado e as formações abertas ocorrem com transformações tênues na estrutura fitossociológica, gerando um brando gradiente fisionômico e a determinação dos fatores responsáveis por essa mudança têm sido alvo de muitas pesquisas. Ribeiro e Walter (2008) relacionam a complexidade dos padrões biogeográficos do bioma com a interação entre os parâmetros bióticos e abióticos que determinam as mudanças quali-quantitativas em suas paisagens.

Segundo Bond (2010), a distribuição biogeográfica dos principais tipos de vegetação regional e global possui geralmente alto grau de associação com o clima. Entretanto, o domínio Cerrado apresenta, em muitos casos, condições climáticas suficientes para o suporte de formações florestais em lugar de savânicas. Neri et al. (2007) salientam que o clima, certamente, é o fator principal para a formação de padrões florísticos e o solo um fator determinante secundário.

Cerne, Lavras, v. 19, n. 2, p. 201-211, abr./jun. 2013
Ruggiero et al. (2002) relatam que a disponibilidade de nutrientes no solo tem sido apontada por muitos autores como um dos determinantes mais importantes do Cerrado. Como peculiaridade, os solos desse bioma em geral apresentam baixa saturação da maioria dos cátions e fósforo necessários ao estabelecimento de vegetação, enquanto os níveis de alumínio são consideravelmente elevados (HARIDASAN, 1982; REATTO et al., 2008; RIBEIRO; WALTER, 2008; RUGGIERO et al., 2002).

As adaptações ecofisiológicas e morfológicas em resposta ao estresse hídrico representam outra peculiaridade da vegetação do Cerrado, indicando que o estresse hídrico inclui-se dentre os determinantes mais importantes dessa vegetação. Essas diversas estratégias entre as espécies lenhosas determinadas por diferentes mecanismos adaptativos permitem compartilhamento de recursos escassos o que reflete na alta biodiversidade de suas comunidades (FELFILI; SILVA JÚNIOR, 2001; HARIDASAN, 2005; RIBEIRO; WALTER, 2008).

De acordo com alguns autores como Goodland e Pollard (1973) e Ribeiro e Walter (2008), há correlação positiva da vegetação de Cerrado e das variáveis edáficas em gradiente de resposta fisionômica na escala regional apresentada, mas esclarecer essa relação em escala de comunidade ainda merece discussão. De tal modo, focando nas propriedades edáficas procuram-se evidências que subsidiem a ideia de um zoneamento da estrutura e da composição de espécies, formando ambientes particulares da comunidade. Diante desse contexto, neste trabalho, objetivou-se verificar a existência de variações na composição e distribuição das espécies arbóreas em virtude das características do solo em um remanescente de cerradão.

\section{MATERIAL E MÉTODOS}

A Fazenda Experimental do Moura (FEM) pertence à Universidade Federal dos Vales do Jequitinhonha e Mucuri (UFVJM), possui uma área contínua de 571 ha, e está localizada no município Curvelo, Minas Gerais $\left(18^{\circ} 45^{\prime} \mathrm{S}\right.$ e $45^{\circ} 25^{\prime} \mathrm{W}$, altitude média de $\left.715 \mathrm{~m}\right)$. A região apresenta clima do tipo $A w$ (clima tropical com inverno seco), segundo a classificação de Köppen, identificado como clima tropical de savana (STRAHLER; STRAHLER, 2002), com temperaturas médias em torno de $28^{\circ} \mathrm{C}$ e índice pluviométrico em torno de $1.200 \mathrm{~mm} / \mathrm{ano}$ (BRASIL, 1992). A vegetação predominante no município de Curvelo é típica de Cerrado. A área de estudo foi um fragmento de cerradão distrófico (RIBEIRO; WALTER, 
2008) de aproximadamente 220,0 ha contínuos com relevo aplainado, pertencente ao grupo fitogeográfico centrosudeste reconhecido por Ratter et al. (2003).

O fragmento é circundado por ambientes antropizados com áreas de pastagem, lagoas artificiais, além de ambientes naturais como uma florestal semidecidual ciliar. A área caracterizou-se por apresentar tanto indivíduos tortuosos com aspectos xeromórficos quanto indivíduos eretos em proporções semelhantes, um dossel quase contínuo na estação chuvosa, formando estratos bem definidos em alguns pontos. Poucas espécies não apresentaram caducifolia no período da estação seca. Há presença rara de bromélias, estrato herbáceo ralo e poucas lianas, além da presença da palmeira arborescente (Syagrus flexuosa (Mart.) Becc.).

Para o presente estudo, foi realizado um inventário florestal por meio de Parcelas Permanentes com esforço amostral de 1,0 ha distribuído em 10 unidades amostrais com dimensões $20 \times 50 \mathrm{~m}\left(1.000 \mathrm{~m}^{2}\right)$ instaladas sistematicamente com distâncias, entre parcelas iguais a $100 \mathrm{~m}$. O critério de inclusão adotado foi o diâmetro ao nível de $0,30 \mathrm{~m}$ do solo (DAS) $\geq 5,0 \mathrm{~cm}$. Indivíduos com caules múltiplos foram incluídos quando a raiz da soma dos quadrados das CAS obedeceu ao critério e foram contabilizados como indivíduo único. Todos os indivíduos vivos foram marcados com etiqueta de alumínio numerada e foram registrados o nome científico, o valor de CAS e a altura total.

A identificação do material botânico foi realizada por meio de consultas à literatura, por especialistas ou por comparações com espécimes existentes nos herbário da UFVJM, segundo o sistema de classificação APG III (ANGIOSPERM PHYLOGENY GROUP - APG, 2009). Todo o material foi identificado, sempre que possível, até o nível de espécie. As exsicatas estão depositadas no Herbário Dendrológico Jeanine Felfili (HDJF), Departamento de Engenharia Florestal (DEF) da UFVJM.

Para descrever a estrutura da comunidade arbórea, foram calculados os parâmetros fitossociológicos clássicos propostos por Mueller-Dombois e Ellemberg (1974). Para avaliar o número total de espécies, foram calculados estimadores jackknife de primeira e segunda ordem, dos quais as projeções a partir da heterogeneidade da amostra foram tomadas como referência (HELTSCHE; FORRESTER, 1983; PALMER, 1991).

Foram coletadas amostras do solo superficial e subsuperficial que formaram duas amostras compostas em cada parcela (0-20 e $20-40 \mathrm{~cm}$ de profundidade).
As análises químicas e granulométricas das amostras foram realizadas no Laboratório de Análise de Solos da UFVJM, seguindo o protocolo da Empresa Brasileira de Pesquisa Agropecuária - EMBRAPA (1997). O ambiente foi caracterizado a partir do resultado das análises de solo. Como uma forma indireta de se estimar a umidade do solo foram tomadas as distâncias entre cada parcela e o curso de água adjacente. Essas distâncias foram calculadas a partir do comprimento do raio cujo círculo é projetado do centro de cada parcela até que seu perímetro tangencie o curso de água, contendo assim um ponto geográfico referente a este (SANTOS et al., 2003).

As correlações entre os gradientes ambientais e vegetacionais foram realizadas pelo emprego de uma análise de correspondência canônica (CCA) (BRAAK, 1987) associada ao teste de Monte Carlo, para verificar a significância dessa análise. As análises multivariadas foram realizadas utilizando-se o programa PC-ORD for Windows versão 4.14 (MCCUNE; MEFFORD, 1999). A matriz de abundância das espécies foi constituída do número de indivíduos (d) por parcela das espécies com densidade superior a cinco indivíduos na amostra. De acordo com as recomendações de Braak (1995), os valores de abundância foram transformados pela expressão $\log _{10}(\mathrm{~d}$ +1) para compensar os desvios causados por alguns poucos valores muito elevados. As variáveis ambientais foram normalizadas, com exceção de valores em percentagem, que foram transformados em arco seno $(\mathrm{x} / 100)$.

\section{RESULTADOS E DISCUSSÃO}

Foram registradas 2.424 indivíduos/ha com uma média de aproximadamente 243 indivíduos/parcela e desvio próximo de 58 indivíduos/parcela. Dentre esses indivíduos foram registradas 92 espécies, sendo três entidades morfologicamente agrupadas e uma identificada somente em nível de gênero. Essa riqueza está distribuída em 36 famílias e 74 gêneros (Tabela 1). Erythroxylum, Byrsonima, Myrcia e Qualea foram os gêneros mais diversos apresentando quatro espécies para o primeiro e três para os demais. Ratter et al. (1997) mencionam a diversidade dos gêneros Erythroxylum e Byrsonima na reserva ecológica da Universidade de Brasília, no Distrito Federal. Tais gêneros também foram citados como mais expressivos em um trecho de cerrado típico na Fazenda Água Limpa-DF (FELFILI et al., 2000). Já, as espécies do gênero Qualea apresentaram acentuada dominância e estão, na literatura, entre os táxons que exprimem o maior percentual da biomassa arbórea e ampla distribuição nos

Cerne, Lavras, v. 19, n. 2, p. 201-211, abr./jun. 2013 
cerrados brasileiros (EITEN, 1974; FELFILI et al., 1994; FELFILI; SILVA-JÚNIOR, 2005; MENDONÇA et al., 2008; RATTER et al., 1996; RIBEIRO et al., 1985).

A riqueza observada neste trabalho é relativamente alta em comparação a trabalhos de outros autores em áreas de Cerrado (BALDUINO et al., 2005; FELFILI et al., 1994,
2000; FELFILI; SILVA-JÚNIOR, 2005), condizendo com a relação de solos distróficos e elevado número de espécies (BRIDGEWATER et al., 2004; RATTER et al., 2003). Os estimadores não paramétricos de jackknife de primeira e segunda ordem projetaram a riqueza de 106,4 e 107,8 espécies, respectivamente.

Tabela 1 - Ordenação decrescente dos valores de IVI das espécies arbóreas registradas e seus parâmetros fitossociológicos em um remanescente de Cerrado da Fazenda do Moura, Curvelo-MG. Em que: $\mathrm{n}_{\mathrm{i}}=$ número de indivíduos; DR = densidade relativa (\%); $\mathrm{FR}=$ freqüência relativa $(\%) ; \mathrm{DoR}=$ dominância relativa $(\%) ; \mathrm{IVI}=$ valor de importância (\%).

Table 1 - Decreasing ordering of woody species according to their phytosociological parameters recorded in a fragment of the Fazenda Experimental do Moura, Curvelo-MG. Whereby: $n_{i}=$ number of individuals; $D R=$ relative density (\%); FR $=$ relative frequency (\%); DoR = relative dominance (\%); IVI = importance value index $(\%)$.

\begin{tabular}{|c|c|c|c|c|c|c|}
\hline Família & Espécies & $\mathrm{n}_{\mathrm{i}}$ & $\mathrm{DR}_{\mathrm{i}}$ & $\mathrm{FR}_{\mathrm{i}}$ & $\operatorname{DoR}_{\mathrm{i}}$ & $\mathrm{IVI}_{\mathrm{i}}$ \\
\hline Sapindaceae & Magonia pubescens A.St.-Hil. & 471 & 19,43 & 2,32 & 13,05 & 11,60 \\
\hline Vochysiaceae & Qualea grandiflora Mart. & 196 & 8,09 & 2,32 & 13,46 & 7,95 \\
\hline Fabaceae & Tachigali subvelutina (Benth.) Oliveira-Filho & 205 & 8,46 & 2,32 & 12,56 & 7,78 \\
\hline Combretaceae & Terminalia argentea (Cambess.) Mart. & 77 & 3,18 & 1,86 & 7,18 & 4,07 \\
\hline Vochysiaceae & Qualea parviflora Mart. & 98 & 4,04 & 2,09 & 4,72 & 3,62 \\
\hline Clusiaceae & Kielmeyera coriacea Mart. \& Zucc. & 90 & 3,71 & 2,09 & 2,32 & 2,71 \\
\hline Burseraceae & Protium heptaphyllum (Aubl.) Marchand & 84 & 3,47 & 1,86 & 2,60 & 2,64 \\
\hline Fabaceae & Leptolobium dasycarpum Vogel & 71 & 2,93 & 2,32 & 2,14 & 2,46 \\
\hline Anacardiaceae & Astronium fraxinifolium Schott ex Spreng. & 51 & 2,10 & 2,32 & 2,01 & 2,14 \\
\hline Fabaceae & Machaerium opacum Vogel & 46 & 1,90 & 2,32 & 1,66 & 1,96 \\
\hline Proteaceae & Roupala montana Aubl. & 45 & 1,86 & 2,09 & 1,58 & 1,84 \\
\hline Fabaceae & Tachigali aurea Tul. & 43 & 1,77 & 1,86 & 1,86 & 1,83 \\
\hline Fabaceae & Vatairea macrocarpa (Benth.) Ducke & 37 & 1,53 & 1,86 & 2,02 & 1,80 \\
\hline Annonaceae & Xylopia aromatica (Lam.) Mart. & 57 & 2,35 & 1,62 & 1,36 & 1,78 \\
\hline Malvaceae & Eriotheca pubescens (Mart. \& Zucc.) Schott \& Endl. & 20 & 0,83 & 1,62 & 2,37 & 1,60 \\
\hline Verbenaceae & Aloysia virgata (Ruiz \& Pav.) A.Juss. & 47 & 1,94 & 1,39 & 1,02 & 1,45 \\
\hline Caryocaraceae & Caryocar brasiliense Cambess. & 12 & 0,50 & 1,62 & 2,19 & 1,44 \\
\hline Bignoniaceae & Tabebuia aurea (Manso) Benth. \& Hook.f. ex S.Moore & 33 & 1,36 & 1,62 & 1,27 & 1,42 \\
\hline Fabaceae & Bowdichia virgilioides Kunth & 16 & 0,66 & 2,09 & 1,38 & 1,38 \\
\hline Dilleniaceae & Curatella americana $\mathrm{L}$. & 16 & 0,66 & 1,62 & 1,82 & 1,37 \\
\hline Erythroxylaceae & Erythroxylum daphnites Mart. & 31 & 1,28 & 2,09 & 0,56 & 1,31 \\
\hline Sapindaceae & Dilodendron bipinnatum Radlk. & 34 & 1,40 & 1,39 & 1,02 & 1,27 \\
\hline Fabaceae & Dimorphandra mollis Benth. & 22 & 0,91 & 2,09 & 0,74 & 1,25 \\
\hline Malvaceae & Pseudobombax tomentosum (Mart. \& Zucc.) A.Robyns & 18 & 0,74 & 1,62 & 1,20 & 1,19 \\
\hline Vochysiaceae & Qualea multiflora Mart. & 23 & 0,95 & 2,09 & 0,44 & 1,16 \\
\hline Malpighiaceae & Byrsonima coccolobifolia Kunth & 27 & 1,11 & 1,39 & 0,87 & 1,12 \\
\hline
\end{tabular}

Cerne, Lavras, v. 19, n. 2, p. 201-211, abr./jun. 2013 
Tabela 1 - Continuação...

Table 1 - Continued...

\begin{tabular}{|c|c|c|c|c|c|c|}
\hline Família & Espécies & $\mathrm{n}_{\mathrm{i}}$ & $\mathrm{DR}_{\mathrm{i}}$ & $\mathrm{FR}_{\mathrm{i}}$ & $\operatorname{DoR}_{\mathrm{i}}$ & $\mathrm{IVI}_{\mathrm{i}}$ \\
\hline Myrtaceae & Eugenia dysenterica DC. & 20 & 0,83 & 2,09 & 0,37 & 1,09 \\
\hline Malpighiaceae & Byrsonima crassa Nied. & 28 & 1,16 & 1,62 & 0,45 & 1,07 \\
\hline Myrtaceae & Myrcia guianensis (Aubl.) DC. & 19 & 0,78 & 1,86 & 0,30 & 0,98 \\
\hline Asteraceae & Piptocarpha rotundifolia (Less.) Baker & 19 & 0,78 & 1,62 & 0,53 & 0,98 \\
\hline Apocynaceae & Himatanthus obovatus (Müll.Arg.) Woodson & 19 & 0,78 & 1,62 & 0,52 & 0,98 \\
\hline Rubiaceae & Cordiera sessilis (Vell.) Kuntze & 19 & 0,78 & 1,39 & 0,74 & 0,97 \\
\hline Apocynaceae & Aspidosperma tomentosum Mart. & 22 & 0,91 & 1,39 & 0,54 & 0,95 \\
\hline Ochnaceae & Ouratea hexasperma (A.St.-Hil.) Baill. & 15 & 0,62 & 1,62 & 0,38 & 0,87 \\
\hline Ebenaceae & Diospyros sericea A.DC. & 23 & 0,95 & 0,70 & 0,98 & 0,87 \\
\hline Erythroxylaceae & Erythroxylum suberosum A.St.-Hil. & 20 & 0,83 & 1,39 & 0,33 & 0,85 \\
\hline Lythraceae & Lafoensia vandelliana Cham. \& Schltdl. & 14 & 0,58 & 1,62 & 0,24 & 0,81 \\
\hline Myrsinaceae & Myrsine gardneriana A.DC. & 13 & 0,54 & 1,62 & 0,20 & 0,79 \\
\hline Annonaceae & Annona crassiflora Mart. & 11 & 0,45 & 1,16 & 0,73 & 0,78 \\
\hline Malpighiaceae & Byrsonima verbascifolia (L.) DC. & 13 & 0,54 & 1,39 & 0,23 & 0,72 \\
\hline Ebenaceae & Diospyros burchellii Hiern. & 20 & 0,83 & 0,93 & 0,39 & 0,72 \\
\hline Celastraceae & Maytenus floribunda Reissek & 22 & 0,91 & 0,70 & 0,49 & 0,70 \\
\hline Malvaceae & Luehea grandiflora Mart. \& Zucc. & 21 & 0,87 & 0,70 & 0,49 & 0,68 \\
\hline Fabaceae & Hymenaea stigonocarpa Mart. ex Hayne & 8 & 0,33 & 1,16 & 0,44 & 0,64 \\
\hline Bignoniaceae & Handroanthus ochraceus (Cham.) Mattos & 6 & 0,25 & 1,16 & 0,49 & 0,63 \\
\hline Ochnaceae & Ouratea castaneifolia (DC.) Engl. & 12 & 0,50 & 1,16 & 0,23 & 0,63 \\
\hline Nyctaginaceae & Guapira noxia (Netto) Lundell & 11 & 0,45 & 0,70 & 0,73 & 0,63 \\
\hline Celastraceae & Plenckia populnea Reissek & 18 & 0,74 & 0,70 & 0,37 & 0,60 \\
\hline Sapotaceae & Pouteria ramiflora (Mart.) Radlk. & 14 & 0,58 & 0,46 & 0,68 & 0,58 \\
\hline Fabaceae & Plathymenia reticulata Benth. & 6 & 0,25 & 0,93 & 0,53 & 0,57 \\
\hline Fabaceae & Copaifera langsdorffii Desf. & 8 & 0,33 & 0,93 & 0,44 & 0,56 \\
\hline Anacardiaceae & Myracrodruon urundeuva Allemão & 8 & 0,33 & 0,93 & 0,32 & 0,53 \\
\hline Rubiaceae & Coussarea hydrangeifolia (Benth.) Müll.Arg. & 8 & 0,33 & 0,93 & 0,31 & 0,52 \\
\hline Bignoniaceae & Zeyheria montana Mart. & 9 & 0,37 & 0,93 & 0,25 & 0,52 \\
\hline Rubiaceae & Guettarda viburnoides Cham. \& Schltdl. & 7 & 0,29 & 1,16 & 0,10 & 0,52 \\
\hline Opiliaceae & Agonandra brasiliensis Miers ex Benth. \& Hook. & 9 & 0,37 & 0,93 & 0,20 & 0,50 \\
\hline Fabaceae & Dalbergia miscolobium Benth. & 4 & 0,17 & 0,70 & 0,56 & 0,47 \\
\hline Meliaceae & Trichilia pallida Sw. & 7 & 0,29 & 0,93 & 0,19 & 0,47 \\
\hline Malpighiaceae & Heteropterys byrsonimifolia A.Juss. & 12 & 0,50 & 0,46 & 0,34 & 0,43 \\
\hline Salicaceae & Casearia sylvestris Sw. & 4 & 0,17 & 0,93 & 0,07 & 0,39 \\
\hline Loganiaceae & Strychnos pseudoquina A.St.-Hil. & 5 & 0,21 & 0,70 & 0,17 & 0,36 \\
\hline
\end{tabular}


Tabela 1 - Continuação...

Table 1 - Continued...

\begin{tabular}{|c|c|c|c|c|c|c|}
\hline Família & Espécies & $\mathrm{n}_{\mathrm{i}}$ & $\mathrm{DR}_{\mathrm{i}}$ & $\mathrm{FR}_{\mathrm{i}}$ & $\operatorname{DoR}_{\mathrm{i}}$ & $\mathrm{IVI}_{\mathrm{i}}$ \\
\hline Rubiaceae & Alibertia edulis (Rich.) A.Rich. ex DC. & 8 & 0,33 & 0,46 & 0,26 & 0,35 \\
\hline Anacardiaceae & Lithraea molleoides (Vell.) Engl. & 10 & 0,41 & 0,46 & 0,15 & 0,34 \\
\hline Rubiaceae & Palicourea rigida Kunth & 4 & 0,17 & 0,70 & 0,08 & 0,31 \\
\hline Fabaceae & Platypodium elegans Vogel & 3 & 0,12 & 0,23 & 0,49 & 0,28 \\
\hline Loganiaceae & Antonia ovata Pohl & 6 & 0,25 & 0,46 & 0,08 & 0,26 \\
\hline Vochysiaceae & Salvertia convallariodora A.St.-Hil. & 3 & 0,12 & 0,46 & 0,18 & 0,25 \\
\hline Bignoniaceae & Handroanthus impetiginosus (Mart. ex DC.) Mattos & 4 & 0,17 & 0,46 & 0,13 & 0,25 \\
\hline Rubiaceae & Rudgea viburnoides (Cham.) Benth. & 4 & 0,17 & 0,46 & 0,11 & 0,25 \\
\hline Nyctaginaceae & Guapira graciliflora (Schmidt) Lundell & 2 & 0,08 & 0,46 & 0,16 & 0,24 \\
\hline Siparunaceae & Siparuna guianensis Aubl. & 4 & 0,17 & 0,46 & 0,04 & 0,22 \\
\hline Myrtaceae & Myrcia neorostrata Sobral & 3 & 0,12 & 0,46 & 0,05 & 0,21 \\
\hline Erythroxylaceae & Erythroxylum tortuosum Mart. & 3 & 0,12 & 0,46 & 0,05 & 0,21 \\
\hline Myrtaceae & Myrcia tomentosa (Aubl.) DC. & 2 & 0,08 & 0,46 & 0,06 & 0,20 \\
\hline Araliaceae & Schefflera macrocarpa (Cham. \& Schltdl.) Frodin & 2 & 0,08 & 0,46 & 0,04 & 0,19 \\
\hline Erythroxylaceae & Erythroxylum deciduum A.St.-Hil. & 2 & 0,08 & 0,46 & 0,03 & 0,19 \\
\hline Fabaceae & Stryphnodendron adstringens (Mart.) Cov. & 2 & 0,08 & 0,46 & 0,02 & 0,19 \\
\hline Bignoniaceae & Tabebuia roseoalba (Ridl.) Sandwith & 3 & 0,12 & 0,23 & 0,07 & 0,14 \\
\hline Moraceae & Brosimum gaudichaudii Trécul & 2 & 0,08 & 0,23 & 0,02 & 0,11 \\
\hline Bignoniaceae & Jacaranda brasiliana (Lam.) Pers. & 1 & 0,04 & 0,23 & 0,06 & 0,11 \\
\hline Asteraceae & Vernonia sp. & 1 & 0,04 & 0,23 & 0,02 & 0,10 \\
\hline Fabaceae & Enterolobium gummiferum (Mart.) J.F.Macbr. & 1 & 0,04 & 0,23 & 0,02 & 0,10 \\
\hline Combretaceae & Buchenavia tomentosa Eichler & 1 & 0,04 & 0,23 & 0,02 & 0,10 \\
\hline Apocynaceae & Aspidosperma subincanum Mart. ex A.DC. & 1 & 0,04 & 0,23 & 0,02 & 0,10 \\
\hline Bignoniaceae & Cybistax antisyphilitica (Mart.) Mart. & 1 & 0,04 & 0,23 & 0,02 & 0,10 \\
\hline Styracaceae & Styrax camporum Pohl & 1 & 0,04 & 0,23 & 0,01 & 0,10 \\
\hline Rubiaceae & Randia aculeata $\mathrm{L}$. & 1 & 0,04 & 0,23 & 0,01 & 0,09 \\
\hline Rubiaceae & Tocoyena formosa (Cham. \& Schltdl.) K.Schum. & 1 & 0,04 & 0,23 & 0,01 & 0,09 \\
\hline Myrtaceae & Calyptranthes lucida Mart. ex DC. & 1 & 0,04 & 0,23 & 0,01 & 0,09 \\
\hline Indeterminadas & & 3 & 0,124 & 0,696 & 0,0339 & 0,28 \\
\hline Total & & 2.424 & 100 & 100 & 100 & 100 \\
\hline
\end{tabular}

Foram encontradas na área fora das parcelas ou não atendendo ao critério de inclusão mais dez espécies (Annona dioica A.St.-Hil., Casearia sp., Davilla elliptica A.St.-Hil., Erythroxylum campestre St. Hil., Eugenia bimarginata DC. (Vahl) S.O.Grose, Heteropterys tomentosa Adr. Juss, Psidium myrsinoides Berg., Savia dictyocarpa Müll. e Styrax camporum Pohl), que adicionadas ao levantamento florístico compilou-se 102 táxons fanerófitos, ainda aquém do valor dado pelos estimadores de jackknife.

As espécies Copaifera langsdorffii e Protium heptaphyllum foram mais expressivas nas parcelas de Borda (marginais à floresta semidecidual). Essas espécies são de dispersão zoocórica, tolerantes à sombra, durante

Cerne, Lavras, v. 19, n. 2, p. 201-211, abr./jun. 2013 
o seu estabelecimento (DURIGAN; RATTER, 2006), podendo ser indicadoras de estabilidade ambiental e avanço sucessional no cerradão, com maior fechamento do dossel e efetividade dos processos ecológicos. Por outro lado, Tachigali subvelutina, Caryocar brasiliense, Byrsonima verbassifolia e Xylopia aromatica foram mais expressivas em parcelas do interior do fragmento, mais distantes da umidade e sob formação mais aberta.

Esse padrão apresentado pode evidenciar um caráter transicional no fragmento. Provavelmente uma relação do gradiente (florístico e estrutural) e da disposição espacial das parcelas, em que, parte sofre influência da zona de maior umidade (menor valor de D na Tabela 2), parte pertence a uma zona de menor umidade (maior valor de D na Tabela 2).

As variáveis ambientais que apresentaram maior correlação interna (com os eixos) foram teor de fósforo $\left(\mathrm{P}_{1}\right)$ e cálcio $\left(\mathrm{C}_{1}\right)$ na camada de 0 a $20 \mathrm{~cm}$ de profundidade do solo e teor potássio $\left(\mathrm{K}_{2}\right)$ e saturação por alumínio $\left(\mathrm{m}_{2}\right)$ na camada de 20 a $40 \mathrm{~cm}$ de profundidade do solo, além da distância das parcelas em relação ao curso de água. Apesar de o solo das parcelas apresentarem baixa fertilidade, com saturação de bases (V\%) inferior a 50\% (distróficos), teores de matéria orgânica (MO) médios e valores muito altos de alumínio (Al) e acidez, segundo Alvarez e Ribeiro (1999), fez-se a ordenação das parcelas, obedecendo a um gradiente de maior fertilidade, considerando as variáveis utilizadas na CCA. Há uma correspondência muito forte entre o número de espécies, área basal ou densidade por parcela e as variáveis ambientais da Tabela 2.

Analisando-se apenas um fator, esses atributos das parcelas tendem positivamente em função do Fósforo (P). Com exceção das parcelas 6, 7 e 8, nessa relação, o número de espécies, área basal e densidade por parcela tendem a ser máximo nos maiores valores de $\mathrm{P}$, e mínimo nos menores. De forma inversa, obtém-se essa ordenação em função da saturação de alumínio (m). As características ambientais apresentaram influência na distribuição de abundância de população, interferindo na estrutura da comunidade. A dificuldade da ordenação perfeita de todas as parcelas possivelmente pode estar relacionada com a não significância da relação espécie ambiente no gradiente sintetizado pelo segundo eixo (Tabela 3 ).

Os resultados da análise de correspondência canônica (CCA) estão apresentados na Tabela 3 e representados no diagrama de ordenação da Figura 1. Verifica-se que a CCA produziu alta correlação espécieambiente nos três primeiros eixos, entretanto pequenos autovalores $(>0,5)$ indicando gradientes curtos, ou seja, as espécies em sua maioria não ocupam setores específicos do ambiente, ou seja, não há grande substituição de espécies ao longo dos eixos, variando, em geral, apenas em abundância em todo o gradiente sintetizado. Os três primeiros eixos sintetizaram uma variação percentual

Tabela 2 - Valores de riqueza, densidade e área basal $\left(\mathrm{AB} \mathrm{m}^{2}\right)$ por parcela, distância do curso de água (D metros) e variáveis dwo solo nas camadas de $0-20 \mathrm{~cm}\left(\mathrm{P}_{1} \mathrm{mg} / \mathrm{dm}^{3}\right.$ e $\left.\mathrm{Ca}_{1} \mathrm{cmol}_{\mathrm{c}} / \mathrm{dm}^{3}\right)$ e $20-40 \mathrm{~cm}\left(\mathrm{~K}_{2} \mathrm{mg} / \mathrm{dm}^{3}\right.$ e $\left.\mathrm{m}_{2} \%\right)$.

Table 2 - Values of richness, density and basal area $\left(A B \mathrm{~m}^{2}\right)$ per plot, distance from the watercourse (D meters) and soil variables in the layers $0-20 \mathrm{~cm}\left(P_{1} \mathrm{mg} / \mathrm{dm}^{3}\right.$ e $\left.\mathrm{Ca}_{1} \mathrm{cmol}_{\mathrm{c}} / \mathrm{dm}^{3}\right)$ and $20-40 \mathrm{~cm}\left(\mathrm{~K}_{2} \mathrm{mg} / \mathrm{dm}^{3}\right.$ e $\left.\mathrm{m}_{2} \%\right)$.

\begin{tabular}{|c|c|c|c|c|c|c|c|c|}
\hline \multirow{2}{*}{ Parcela } & \multirow{2}{*}{$\mathrm{N}^{\circ}$ Espécies } & \multirow{2}{*}{$\mathrm{N}^{\mathrm{o}}$ Indivíduos } & \multirow{2}{*}{$\mathrm{AB}$} & \multirow{2}{*}{$\mathrm{D}$} & $\mathrm{P}$ & $\mathrm{Ca}$ & $\mathrm{K}$ & $\mathrm{m}$ \\
\hline & & & & & \multicolumn{2}{|c|}{$0-20 \mathrm{~cm}$} & \multicolumn{2}{|c|}{$20-40 \mathrm{~cm}$} \\
\hline 1 & 55 & 328 & 2,75 & 117 & 1,9 & 0,7 & 53,0 & 65,9 \\
\hline 2 & 41 & 305 & 2,48 & 129 & 1,4 & 0,8 & 53,0 & 67,9 \\
\hline 3 & 40 & 220 & 2,39 & 144 & 1,3 & 0,6 & 51,0 & 72,9 \\
\hline 4 & 35 & 167 & 1,50 & 195 & 0,9 & 0,6 & 54,0 & 66,9 \\
\hline 5 & 39 & 229 & 2,41 & 206 & 0,9 & 0,6 & 51,0 & 75,9 \\
\hline 6 & 47 & 265 & 2,45 & 124 & 1,4 & 0,6 & 51,0 & 72,1 \\
\hline 7 & 45 & 314 & 2,65 & 162 & 0,6 & 0,5 & 54,0 & 72,4 \\
\hline 8 & 50 & 213 & 2,22 & 147 & 1,0 & 0,6 & 47,0 & 73,3 \\
\hline 9 & 40 & 213 & 2,07 & 233 & 1,0 & 0,5 & 32,0 & 80,5 \\
\hline 10 & 39 & 170 & 1,86 & 253 & 1,2 & 0,6 & 32,0 & 81,1 \\
\hline Média & 43,1 & 242,4 & 2,2775 & & & & & \\
\hline
\end{tabular}

Cerne, Lavras, v. 19, n. 2, p. 201-211, abr./jun. 2013 
Tabela 3 - Resumo dos resultados das análises de correspondência canônica da abundância de espécies em interação com variáveis ambientais e dos testes de permutação de Monte Carlo correspondentes. São fornecidos resultados para os três primeiros eixos de ordenação.

Table 3 - Summary of canonical correspondence analyses of the abundance of species in interaction with environmental and spatial variables, and the corresponding Monte Carlo permutation tests. Results are given for the first three ordination axes.

\begin{tabular}{llccc}
\hline & \multirow{2}{*}{ CCA } & & \multicolumn{2}{c}{ Eixos } \\
\cline { 3 - 5 } Autovalores & & 1 & 2 & 3 \\
\hline Variância percentual (\%) de: & Dados & 0,174 & 0,114 & 0,073 \\
& Cumulativa dos dados & 24,2 & 15,8 & 10,2 \\
Correlação de Pearson - espécie $\times$ ambiente & & 24,2 & 39,9 & 50,1 \\
\hline Testes de permutação de Monte Carlo: & Autovalores & 0,996 & 0,969 & 0,989 \\
& Correlação espécie ambiente & $0,001^{* *}$ & $0,013^{*}$ & 0,10 \\
\hline Correlações internas com os eixos (r): & Distância do Curso d'água & $0,029^{*}$ & 0,18 & $0,04^{*}$ \\
& Fósforo (P1) & -0.613 & -0.240 & 0.616 \\
& Cálcio (Ca1) & 0.545 & -0.555 & -0.577 \\
& Potássio (K2) & 0.130 & -0.055 & -0.927 \\
& Saturação por Alumínio (m2) & 0.566 & 0.712 & -0.352 \\
& & -0.499 & -0.404 & 0.563 \\
\hline
\end{tabular}

$(*)$ e $(* *)$ são valores significativos a $95 \%$ e $99 \%$ de probabilidade respectivamente.

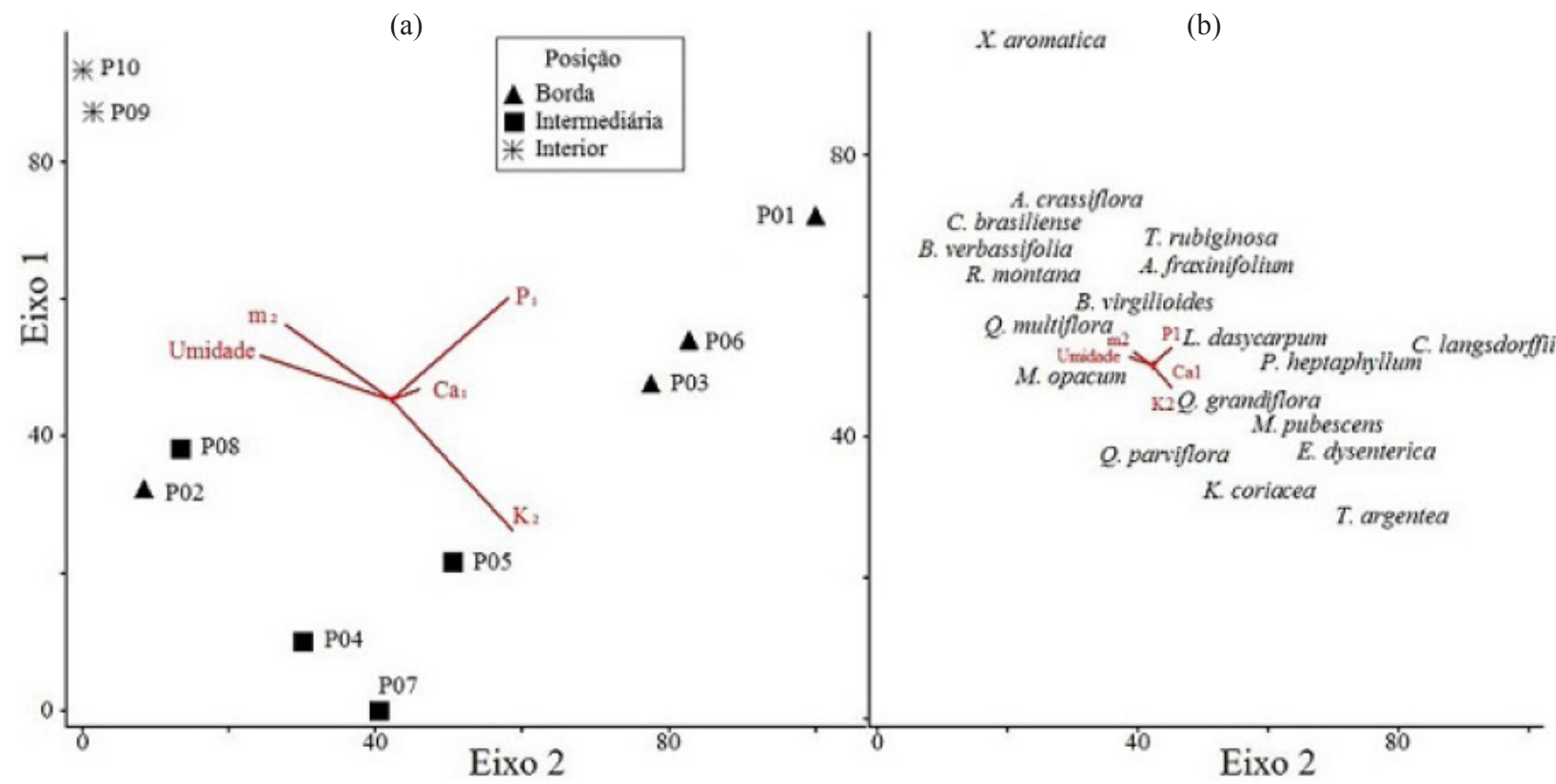

Figura 1 - Diagramas de ordenação a) das parcelas e b) das espécies, produzidos pela análise de correspondência (CCA) da distribuição do número de indivíduos amostrados em dez parcelas de um fragmento de cerradão na Fazenda do Moura, Curvelo-MG.

Figura 1 - Ordination diagram a) of plots and b) of species, produced by canonical correspondence analysis (CCA) of distribution of the number of individuals sampled in ten plots of a fragment of cerradão in Fazenda Experimental do Moura, Curvelo- $M G$.

Cerne, Lavras, v. 19, n. 2, p. 201-211, abr./jun. 2013 
expressiva dos dados de vegetação (cerca de 50\%) explicada pelo ambiente (BRAAK, 1995). Os testes de Monte Carlo indicaram diferenças significativas entre as correlações encontradas e a que pode ser produzida pelo acaso para o primeiro e terceiro eixo. De forma geral, as parcelas se ordenaram quanto à categoria de Borda (marginal à florestal semidecidual), Intermediária e Interior do Fragmento, restando, apenas, à parcela 2 um padrão divergente.

O diagrama da CCA confirmou o gradiente florístico em virtude da tênue variação de fertilidade do solo. Esse gradiente pode ser exemplificado pela densidade apresentada por Magonia pubescens, Terminalia argentea e Protium heptaphyllum, três espécies associadas a solos mais férteis em áreas de Cerrado (ALLEM; VALLS, 1987; BRIDGEWATER et al., 2004; RATTER et al., 2002, 2003).

A espécie M. pubescens foi presente em todas as parcelas, mas sob um forte decréscimo no extremo desse gradiente de fertilidade (parcelas 9 e 10 - Figura 1b) apresentando uma amplitude de 95 indivíduos/parcelas. De forma bastante similar, a população de Terminalia argentea distribui-se sob o mesmo gradiente apresentandose decrescente com a redução da fertilidade.

Em uma visualização direcionada a três populações (Annona crassiflora, Eugenia dysenterica e M. pubescens) foi possível descrever um padrão dentro da comunidade de acordo com a literatura. Pelo diagrama de ordenação (Figura 1b), M. pubescens e E. dysenterica ocuparam um setor oposto a A. crassiflora e Xylopia aromática que tiveram suas populações, principalmente relacionadas a solos de menor fertilidade e maior acidez.

Um padrão similar de distribuição entre $A$. crassiflora e E. dysenterica em função da fertilidade do solo foi também evidenciado no Estado de Goiás por Naves et al. (1995). Esses autores relacionam E. dysenterica a solos em situação intermediária de fertilidade natural, pela plasticidade da espécie. Por outro lado, os mesmos autores relacionam A. crassiflora a solos de mais baixa fertilidade e elevada acidez, corroborando com os resultados aqui apresentados. Já, para Xylopia aromatica um resultado similar ao obtido no presente estudo foi observado por Ruggiero et al. (2002), em que as variações populacionais dessa espécie foram relacionadas aos altos teores de troca alumínio no solo.

\section{CONCLUSÕES}

Verificou-se que houve variações na distribuição das espécies arbóreas em função das variações na distância de um curso de água, da fertilidade ( $\mathrm{P}, \mathrm{K}$ e $\mathrm{Ca}$ ) e da acidez do solo.

\section{REFERÊNCIAS}

ALLEM, A. C.; VALLS, J. F. M. Recursos forrageiros do Pantanal Mato-Grossense. Brasília: EMBRAPA-CENARGEN, 1987. 339 p. (Documentos, 8).

ALVAREZ, V. H.; RIBEIRO, A. C. Calagem. In: RIBEIRO, A. C.; GUimarães, P. T. G.; AlvareZ, V. H. (Ed.). Recomendações para o uso de corretivos e fertilizantes em Minas Gerais. Viçosa, MG: CFSEMG, 1999. p. 43-60.

ANGIOSPERM PHYLOGENY GROUP. An update of the Angiosperm Phylogeny Group classification for the orders and families of flowering plants: APG III. Botanical Journal of the Linnean Society, London, v. 161, p. 105-121, 2009.

BALDUINO, A. P. C.; SOUZA, A. L.; SILVA, A. F.; SILVA JÚNIOR, M. C. Fitossociologia e análise comparativa da composição florística do Cerrado da flora de Paraopeba, MG. Revista Árvore, Viçosa, v. 29, n. 1, p. 25-34, jan./ fev. 2005.

BOND, W. J. Do nutrient-poor soils inhibit development of forests?: a nutrient stock analysis. Plant and Soil, The Hague, v. 334, n. 1/2, p. 47-60, 2010.

BOURLIÈRE, F.; HADLEY, M. Present-day savannas: an overview. In: BOURLIÈRE, F. (Ed.). Tropical Savannas. Oxford: Elsevier, 1983. p. 1-17.

BRAAK, C. J. F. ter. Analysis of vegetation-environment relationships by canonical correspondence analysis. Vegetation, Knivsta, v. 69, p. 69-77, 1987.

BRAAK, C. J. F. ter. Ordination. In: JONGMAN, R. H. G.; BRAAK, C. J. F. ter; TONGEREN, O. F. R. van (Ed.). Data analysis in community and landscape ecology. Cambridge: Cambridge University, 1995. p. 91-173.

BRASIL. Ministério da Agricultura e Reforma Agrária. Normais climatológicas de 1961 - 1990. Brasília, 1992. 84 p.

BRIDGEWATER, S.; RATTER, J. A.; RIBEIRO, J. F. Biogeographic patterns, b-diversity and dominance in the Cerrado biome of Brazil. Biodiversity and Conservation, London, v. 13, p. 2295-2318, 2004.

Cerne, Lavras, v. 19, n. 2, p. 201-211, abr./jun. 2013 
COUTINHO, L. M. O conceito de Cerrado. Revista Brasileira de Botânica, São Paulo, v. 1, p. 17-23, 1978.

DURIGAN, G.; RATTER, J. A. Successional changes in Cerrado and Cerrado/forest ecotonal vegetation in western São Paulo State, Brazil, 1962-2000. Edinburgh Journal of Botany, Edinburgh, v. 63, p. 119-130, 2006.

EITEN, G. Brazilian "savannas". In: HUNTLEY, B. J.; WALKER, B. H. (Ed.). Ecology of Tropical Savannas. New York: Springer-Verlag, 1982. p. 25-47. (Ecological, Studies, 42).

EITEN, G. Outline of the vegetation of South America. In: CONGRESS OF THE INTERNATIONAL PRIMATOLOGICAL SOCIETY, 1., 1974, Nagoya. Proceedings... Nagoya, 1974. p. 529-545.

EMPRESA BRASILEIRA DE PESQUISAAGROPECUÁRIA. Centro Nacional de Pesquisa de Solos. Manual de métodos de análise de solo. 2. ed. Rio de Janeiro, 1997. 212 p.

FELFILI, J. M.; FILGUEIRAS, T. S.; HARIDASAN, M.; SILVA JÚNIOR, M. C.; MENDONÇA, R.; REZENDE, A. V. Projeto biogeografia do bioma Cerrado: vegetação e solos. Cadernos de Geociências do IBGE, Rio de Janeiro, v. 12, p. 75-166, 1994.

FELFILI, J. M.; REZENDE, A. V.; SILVA JÚNIOR, M. C; SILVA, M. A. Changes in the floristic composition of Cerrado sensu stricto in Brazil over a nine-year period. Journal of Tropical Ecology, Cambridge, v. 16, p. 579-590, 2000.

FELFILI, J. M.; SILVA JÚNIOR, M. C. Biogeografia do bioma Cerrado: estudo fitofisionômico na Chapada do espigão Mestre do São Francisco. Brasília: UnB, 2001. 152 p.

FELFILI, J. M.; SILVA JÚNIOR, M. C. Diversidade alfa e beta no Cerrado sensu stricto, Distrito Federal, Goiás, Minas Gerais e Bahia. In: SCARIOT, A.; SOUSA-SILVA, J. C.; FELFILI, J. M. (Org.). Cerrado: ecologia, biodiversidade e conservação. Brasília: Ministério do Meio Ambiente, 2005. p. 143-154.

GOODLAND, R.; POLLARD, R. The Brazilian Cerrado vegetation: a fertility gradient. Journal of Ecology, Oxford, v. 61, p. 219-224, 1973.

HARIDASAN, M. Aluminium accumulation by some Cerrado native species of central Brazil. Plant and Soil, The Hague, v. 65, p. 265-273, 1982.

Cerne, Lavras, v. 19, n. 2, p. 201-211, abr./jun. 2013
HARIDASAN, M. Competição por nutrientes em espécies arbóreas do Cerrado. In: SCARIOT, A.; FELFILI, J. M.; SOUSA-SILVA, J. C. (Org.). Cerrado: ecologia, biodiversidade e conservação. Brasília: Ministério do Meio Ambiente, 2005. p. 167-178.

HELTSCHE, J. F.; FORRESTER, N. E. Estimating species richness using the jackknife procedure. Biometrics, Washington, v. 39, n. 1, p. 1-12, 1983.

MCCUNE, B.; MEFFORD, M. J. PC-ORD multivariate analysis of ecological data: users guide. Version 4.0. Glaneden Beach: MjM Software Design, 1999. 148 p.

MENDONÇA, R. C.; FELFILI, J. M.; WALTER, B. M. T.; SILVA JÚNIOR, M. C. da; REZENDE, A. V.; FILGUEIRAS, T. S.; NOGUEIRA, P. E. Flora vascular do Cerrado. In: SANO, S. M.; ALMEIDA, S. P. (Ed.). Cerrado: ecologia e flora. Planaltina: EMBRAPA, 2008. p. 289-556.

MUELLER-DOMBOIS, D.; ELLENBERG, H. Aims and methods of vegetation ecology. New York: J. Wiley, 1974. $547 \mathrm{p}$.

NAVES, R. V.; ALMEIDA NETO, J. X.; ROCHA, M. R.; BORGES, J. D.; CARVAlHO, G. C.; CHAVES, L. J. Determinação de características físicas em frutos e teor de nutrientes em folhas e no solo, de três espécies frutíferas de ocorrência natural nos Cerrados de Goiás. Anais da Escola de Agronomia e Veterinária, Goiânia, v. 25, p. 99-106, 1995.

NERI, A. V.; MEIRA NETO, J. A. A.; SILVA, A. F.; MARTINS, S. V.; SAPORETI JÚNIOR, A. W. Composição florísitca de uma área de cerrado sensu stricto no município de Senador Modestino Gonçalves, Vale do Jequitinhonha (MG) e análise de similaridade florística de algumas áreas de Cerrado em Minas Gerais. Revista Árvore, Viçosa, v. 31, n. 6, p. 123-134, nov./ dez. 2007.

OLIVEIRA-FILHO, A. T.; RATTER, J. A. Vegetation physiognomies and woody flora of the cerrado biome. In: OLIVEIRA, P. S.; MARQUIS, R. J. (Ed.). The Cerrados of Brazil. New York: Columbia University, 2002. p. 91 120 .

PALMER, M. W. Estimating species richness: the second-order jackknife estimator reconsidered. Ecology, Durham, v. 72, n. 4, p. 1512-1513, 1991. 
RATTER, J. A.; BRIDGEWATER, S.; ATINKINSON, R.; RIBEIRO, J. F. Analysis of the floristic composition of the Brazilian Cerrado vegetation II: comparison of the wood vegetation of 98 areas. Edinburgh Journal of Botany, Edinburgh, v. 53, p. 153-180, 1996.

RATTER, J. A.; BRIDGEWATER, S.; RIBEIRO, J. F. Analysis of the floristic composition of the Brazilian Cerrado vegetation III: comparision of the woody vegetation of 376 areas. Edinburgh Journal of Botany, Edinburgh, v. 60, p. 57-109, 2003.

RATTER, J. A.; RIBEIRO, J. F.; BRIDGEWATER, S. The Brazilian Cerrado vegetation and threats to its biodiversity. Annals of Botany, London, v. 80, p. 223-230, 1997.

REATTO, A.; CORREIA, J. R.; SPERA, S. T.; MARTINS, E. S. Solos do bioma Cerrado: aspectos pedológicos. In: SANO, S. M.; ALMEIDA, S. P.; RIBEIRO, J. F. (Ed.). Cerrado: ecologia e flora. Planaltina: EMBRAPA Cerrados, 2008. v. 1, p. 107-134.

RIBEIRO, J. F.; SILVA, J. C. S.; BATMANIAN, G. J. Fitossociologia de tipos fisionômicos de Cerrado em Planaltina,
DF. Revista Brasileira de Botânica, São Paulo, v. 8, p. 131$142,1985$.

RIBEIRO, J. F.; WALTER, B. M. T. Fitofisionomias do Cerrado. In: SANO, S.; ALMEIDA, S. (Ed.). Cerrado: ecologia e flora. Planaltina: EMBRAPA-CPAC, 2008. p. 89-166.

RUGGiero, P. G. C.; BATALHA, M. A.; PIVELlO, V. R.; MEIRELLES, S. T. Soil-vegetation relationships in Cerrado (Brazilian savanna) and semideciduous forest, Southeastern Brazil. Plant Ecology, Dordrecht, v. 169, p. 161-176, 2002.

SANTOS, F. D. B. E.; FONTES, M. A. L.; OLIVEIRA FILHO, A. T.; BOTEZELLI, L. Análise das variações florísticas e estruturais da comunidade arbórea de um fragmento de floresta semidecídua às margens do rio Capivari, Lavras, MG. Revista Árvore, Viçosa, v. 27, n. 2, p. 185-206, mar./abr. 2003.

STRAHLER, A.; STRAHLER, A. N. Physical geography: science and systems of the human environment. $2^{\text {nd }}$ ed. New York: J. Wiley, 2002. 748 p.

Cerne, Lavras, v. 19, n. 2, p. 201-211, abr./jun. 2013 
